\title{
Option Pricing in Incomplete Markets
}

\author{
Zhaohai WANG \\ Department of mathematics and statistics, Ankang University, Ankang, Shanxi, 725000, china \\ akwzh@163.com
}

\begin{abstract}
If the market is incomplete, we have several choices of equivalent martingale measures to price contingent claims .Recall that we didn't impose conditions on the preferences of the economic agents other than that they prefer more to less.

So it is quite natural to specify the preferences further in order to select one of the equivalent martingale measures. The specification of the investor's attitude towards risk is done in terms of a utility function.
\end{abstract}

Index Terms - Option, Credit risk, Martingale, Risk neutrality

\section{Introduction}

Credit risk is increasingly being recognised as a significant area of risk and arbitrage, yet there exists relatively little research on it. In this paper we show that operational risk represents a fundamental risk to option hedging [1] and investigate it by proposing a new theoretical model. We derive an exposure indicator for the operational risk of option hedging and the resulting operational risk distribution. We obtain analytical results for various risk measures including the Value at Risk equation; this includes deriving a new analytical result for the option function of the half-normal distributions (which will be of interest to Statisticians in general). We determine an analytical solution to the price of options under operational risk [2]. We conduct numerical experiments on empirical option data to validate our model and estimate the operational Value at Risk for option hedging.

There exists currently a good deal of literature on hedging methods for the pricing options. It is well known that in this case a straightforward Monte Carlo simulation algorithm will be time-consuming and yield unstable results for the prices and especially the sensitivities. The knock-in/out features in the barrier option payoffs lead to slower convergence of the Monte Carlo algorithm. To address this problem the following (semi-)analytical approaches have been developed for specific models.

In any case, theoretically, long-run uncertainty about demographic changes and shocks restricts the land supply and raises the land price. The consequences for housingpolicies may be significant, especially in countries (likeFrance) where policymakers attempt to develop the housingstock and to make housing more affordable for the less well-off. Now, the spatial demographic risk is local by nature: a shortfall in one place entails a surplus somewhere else; therefore, it is an insurable risk. Consequently, a future insurance market may be a tool with which to improve the way the housing market works.
Empirically, we conduct the analysis for a French department, Nord. The data base used in this study is made up of individual transactions for developable land for residential purposes(19,495observations) or secondary or tertiary activities (1,667 observations) between 1989 and 2002.The results show that, during the upward trend in the land market, the price of developable land is significantly higher when the change in population in the vicinity is more volatile, and so more difficult to predict. The plot price is $7.8 \%$ (housing market) or $15.3 \%$ (office and factory market) higher when population change in the 10 nearest communes increases by one standard deviation.

\section{A General Option-Pricing Formula}

we compare our results with those given by Merton [3].We first explain Merton's results. Merton restricted his discussion to discrete probability distributions. Let an asset have terminal value $x_{i}$ with probability $f\left(x_{i}\right)$, where the values of $x_{i}$ with positive probability are $x_{1}<x_{2}<\cdots x_{m}$.In addition to the two well-known definitions of increase in risk, namely, a meanpreserving spread and Rothschild and Stiglitz increase in risk, he pro-posed two more definitions of changes in risk which are called pointwise riskier and extremum riskier, respectively. The following are the two definitions.

Definition 2 (Merton.[3]): A distribution $\mathrm{g}(\mathrm{x})$ is said to be pointwise riskier than distribution $\mathrm{f}(\mathrm{x})$ if $\mathrm{s}(\mathrm{x}) \equiv \mathrm{g}(\mathrm{x})-\mathrm{f}(\mathrm{x})$ is a mean-preserving spread whosesupport contains the support of the original distribution $\mathrm{f}(\mathrm{x})$.

Definition3(Merton[3]): Adistribution $\mathrm{g}(\mathrm{x})$ is said to be extremum riskier than distribution $\mathrm{f}(\mathrm{x})$ if the cumulative distribution $\mathrm{G}(\mathrm{x})$ is strictly larger than $\mathrm{F}(\mathrm{x})$ at the immediate right of the left border of the support of $\mathrm{f}(\mathrm{x})$ and is strictly smaller than $\mathrm{F}(\mathrm{x})$ at the immediate left of the right border of the support of $\mathrm{f}(\mathrm{x})$. Rasmusen's Proposition 1 states that a mean-preserving increase in risk does not reduce any option values, which is Merton's (1973) Theorem .His Proposition 1a states that a mean-preserving increase in risk strictly increases all option values, which he proved to be false. His Proposition $1 \mathrm{~b}$ states that a mean-preserving increase in risk strictly increases some option values and reduces no option values. These two results are relatively straightforward. Thus, we focus on his main results.

Foundation items: Natural Scicence Foundation of Ankang Univercity (No, 2013AYPYZR03 )

About the author: Zhaohai wang( $1966-)$, Associate Professor, Research direction: Option Pricing; Optimization; Fuzzy reasoning; Risk Analysis. 


\section{Hedging in Incomplete Markets}

\subsection{The option pricing with risk neutrality argument}

We would like to present an alternative approach of deriving the Black-Scholes equation for the option pricing model, by which the argument of risk neutrality can be explained in a more succinct manner (Cox and Ross [7], 1976). Suppose we write the stochastic process followed by the option price as

$$
\frac{d c}{c}=\mu d t+\sigma d Z
$$

Where $\mu$ is the expected rate of return of $\mathrm{c}$ and $\sigma^{2}$ the corresponding variance of the rate of return .

$$
d c=\left(\frac{\partial c}{\partial t}+\mu S \frac{\partial c}{\partial S}+\frac{1}{2} \frac{\partial^{2} c}{\partial S^{2}} \sigma^{2} S^{2}\right) d t+\sigma S \frac{\partial c}{\partial S} d Z
$$

The above statement of risk neutrality can be represented mathematically as $C=C(S, t)=e^{-r \tau} E\left[\max \left(S_{T}-K, 0\right)\right]$. The option pricing model takes the following form

$$
\begin{aligned}
& C=S N\left(d_{1}\right)-K e^{-r \tau} N\left(d_{2}\right) \quad \text { Where } \quad d_{1}=\frac{\ln (S / K)+\left(r+\frac{\sigma^{2}}{2}\right) T}{\sigma \sqrt{T}}, \\
& d_{2}=d_{1}-\sigma \sqrt{\tau}=\frac{\ln (S / K)+\left(r-\frac{\sigma^{2}}{2}\right) \tau}{\sigma \sqrt{\tau}}, \tau=T-t, \sigma=\sqrt{\operatorname{var}(d S / S)} .
\end{aligned}
$$

The above call price formula can be interpreted using the language of probability. First , $N\left(d_{2}\right)$ is been as the probability of the call option being in-the-money at expiry and so $K N\left(d_{2}\right)$ can be interpreted as the risk neutral expectation of the payment made by the holder of the call option at expiry on exercising the option. Hence, the expectation of the call value at expiry is

$$
S e^{r \tau} N\left(d_{1}\right)-K N\left(d_{2}\right)
$$

which is then discounted by the factor $e^{-r \tau}$ in the risk neutral world to give the present value of the call price.

\subsection{The option pricing in Incomplete Markets}

To model short-term option values [4](temporary price volatility) we look at 'pure' price variability over time, that is, by controlling a set of variables $\mathrm{X}$ that affect this price and vary over the course of time. The starting equation is $d p=\alpha_{1} d t+\sigma \varepsilon_{t} \sqrt{d t}$, where $\mathrm{P}$ is the change in the price of developable land over the period dt under the 'pure' influence of time, that is, having expurgated factors of variation included in $\mathrm{X}$ and where $\varepsilon$, is a random variable of zero mean and of unit variance; $\alpha$ is the trend and $\sigma^{2}$ the price variance. $\mathrm{P}$ is estimated by a random-effects model

$$
P_{i j t}=X_{i j t} b+b_{t} T+v_{t}+\varepsilon_{j}+\varepsilon_{i j t}
$$

where the price $P_{i j t}$ of transaction $\mathrm{i}$ in commune $\mathrm{j}$ and in the period $\mathrm{t}$ is explained by a set of variables $\mathrm{X}$, by a continuous time variable $\mathrm{T}$ capturing the trend, by a random variable $v_{t}$ that is dependent on the period preceding $t$, a second random variable depending on the commune $\mathrm{j}$ and by an individual error $\varepsilon_{i j t}$. The variance of $v_{t}$ is then introduced into the explanatory model of land price where $\sigma_{n}$ is the standard deviation of variation of population change in the communes around $\mathrm{j}$ within a neighborhood $\mathrm{w}$ and $\Delta_{n}$ is the change in population over this period. This standard deviation is calculated directly from population censuses. The standard deviation of population on which households' base their decision to purchase has to be observed over a long enough period.

\section{Option Pricing Model Analysis}

In this paper, we presented an algorithm for pricing barrier options in one-dimensional Markovian models based on an approximation by continuous-time Markov chains. The generator of the approximating chain is constructed by the matching of instantaneous moments of the infinitesimal generator of the Markov process in question, on a suitable nonuniform grid. The approximate barrier option prices are then obtained by calculating the corresponding first-passage distributions for the approximating Markovchain. To illustrate the flexibility of the method we implemented the algorithm for a numberof models, including local volatility models with jumps and models with time-dependent jump-distributions. In the cases of the diffusion and jump-diffusion models where results had been obtained before in the literature, the algorithm produced outcomes that accurately matched those results, and we numerically investigated the order of decay of the error.

We derived a theoretical upper bound for the error of the outcomes produced by the algorithm that is linear in the spatial mesh size and the truncation error. We showed that an additional logarithmic factor may arise in this error bound when the Lévy density has a pole of order two at the origin. In addition, this bound is also linear in the time mesh size if the model is time-inhomogeneous. Numerical experiments suggest that for a number of models the error of the outcomes generated by the algorithm actually decays quadratically in the spatial mesh size. Itwould be of interest to establish error bounds under weaker regularity assumptions, andobtain sharp rates of convergence for the specific models, which is a topic left for future research[5]. Although in principle the method also applies to higher-dimensional Markov processes, the size of the generator matrix would make straightforward application of the algorithm computationally infeasible. The investigation of efficient extensions of the approach to Markov processes of moderate dimension is another topic left for future research. 


\section{Conclusions}

The price of Incomplete Markets follows a geometric Brownian motion in the Black-Scholes model. If the finance market is complete this paper gives an accurate hedging strategy by another method. Then we introduce a dynamic measure of risk to the incomplete market, under which we have acquired the optimal replication of a contingent claim in the finance market which is induced by a risk neutral probability measare. With an application of a generalized Clark formula[6] the paper provides the optimal hedging strategy for a contingent claim. The preferred spelling of the word "acknowledgment" in America is without an "e" after the "g.".

\section{References}

[1] F.Black, M.Scholes. The pricing of options and corporate liabilities. Journal of Political Economy, vol. 81, pp.637-659, April 1973.

[2] Merton R.Theory of rational option pricing. Bell Journal of Economics, vol.4, pp.141-183, 1973.

[3] R.C.Merton. On the Pricing of Corporate Debt: The Risk Structure of Interest Rate. Journal of Finance, vol.29, pp. 449-470, 1974.

[4] H.Johnson, RStulz. The Pricing of options with default risk. Journal of Finance, vol.42, pp.267-280, 1987.

[5] R. A.Jarrow, S.Turnbulll. Pricing derivatives on financial securities subject to credit risk .Journal of Finance, 1995, pp. 53-86.

[6] L.T. Bulan, C..Mayer, C.T. Somerville. Irreversible investment, real options, and competition: evidence from real estate development. JUrban Econ; Vol.65, pp. 237-51, 2009. 